Bioscientia Medicina: Journal of Biomedicine \& Translational Research

Journal Homepage: www.bioscmed.com

\title{
Microneedling Radiofrequency for Acne Vulgaris and Post-Acne Scarring: A Case Report
}

\section{Vesri Yossy ${ }^{1}$, Satya Wydya Yenny ${ }^{2 *}$, Lilik Norawati ${ }^{3}$}

1,2 Department of Dermatology and Venereology, Faculty of Medicine, Andalas University/ Dr. M. Djamil Hospital, Padang, Indonesia

${ }^{3}$ Kartika Estetika Cosmetic Dermatology Clinic Presidential- Army Central Hospital Gatot Soebroto, Jakarta, Indonesia

\section{A R T I C L E I N F O \\ Keywords: \\ Acne vulgaris \\ Radiofrequency micronneedling \\ Post acne scarring \\ *Corresponding author: \\ Satya Wydya Yenny \\ E-mail address: \\ satyawidyayenny@med.unand.ac.id}

All authors have reviewed and approved the final version of the manuscript.

https://doi.org/10.37275/bsm.v6i3.456

\begin{abstract}
A B S T R A C T
Background. There are various therapeutic modalities for acne and post acne scarring, but the best option is to have a safe, effective with affordable cost such as monotherapy or combination therapy. One of the recommended combination therapy for acne vulgaris with atrophic acne scars is microneedling radiofrequency which can improve skin structure. Case presentation: A 25 years old male with moderate comedonal acne vulgaris and severe scarring. The patient came with complaints of uneven skin surface on both cheeks, forehead and chin which had been felt to increase since 3 years ago. The appearance of acne on the face has been felt since 12 years ago. The patient's parents and sister also had acne complaints. On dermatological examination, on the forehead, temples, cheeks, nose and chin found blackheads, whiteheads, papules, hyperpigmented macules, multiple atrophic acne scars. The treatment choice for this patient is microneedling radiofrequency in combination with topical therapy. Discussion: The combination of microneedling with radiofrequency is one of the therapeutic modalities for acne vulgaris and also post-acne scarring with minimal side effects. In the evaluation for 2 weeks after the procedure, this patient did not complaint of any adverse events.
\end{abstract}

\section{Introduction}

Post-acne scar treatment options are chemical peels, spot TCA peels, laser resurfacing, dermabrasion, ablative $\mathrm{CO} 2$ lasers, fillers, subsides and microneedling. Microneedling radiofrequency can cause injury to reach the dermis which increases collagen deposition in scar therapy. Microneedling radiofrequency can reduce the degree of acne and reduce acne lesions seen with a decrease in inflammatory markers (NF-kB and IL-8) in the current study. Inflammation is one of the main pathogenesis of acne microneedling radiofrequency may play a role in the clinical improvement of this skin disorder. The degree of acne scarring is related to the severity, duration of acne and previous treatment. The microneedling radiofrequency procedure helps the physiological wound healing process and stimulates natural collagen production with minimally invasive treatments. $1,2,3$

\section{Case Presentation}

A male 25 years old came to the clinic with chief complaints of uneven skin surface due to acne scars 
that do not feel itchy and painful on the forehead, left and right temples, left and right cheeks and chin since 3 years ago. At first 12 years ago, pimples started to appear in the form of small red spots on both cheeks. The patient often holds and squeezes the pimple on his face, sometimes bleeding. Patients feel disturbed by the complaint of acne scars. The uneven skin surface on the forehead, left and right temples, left and right cheeks, nose and chin has increased since 3 years ago. The patient's sister also has acne and his parents also has acne scars on the face. \pm 6 months ago, the patient went to a Dermatologist and Venereologist for complaints of acne and acne scars. The patient received a night cream $(0.025 \%$ tretinoin cream and $1.2 \%$ clindamycin gel), a morning ointment $(1.2 \%$ clindamycin gel used in the morning on acne). The patient had done microneedling 1 month ago. Acne is felt to be reduced and not many new pimples appear. There is a slightly improvement in the acne scars.
Physical examination on facial regio revealed blackheads (25), whiteheads (12), papules (24) with total number of lesions are 61, patient was diagnosed with moderate comedonal acne based on the clinical severity by Lehman. We also found hyperpigmented macules, multiple atrophic scars with varying shapes, including icepick, rolling, and boxcar scars. Scars disappear on stretching distributed over the forehead, face, nose and chin. Based on the qualitative analysis Goodmans global scarring grading system, the patient's acne scars were grade 3 (moderate). Qualitative analysis was used to assess the severity of acne scars. Quantitative analysis got a score of 19 . We choose microneedling radiofrequency to treat acne scar and a topical medication after procedure. Patient is suggested to continue his acne medication 3 days after procedure with tretinoin 0,025\% cream, clindamycin gel 1,2\% at night and sunscreen SPF 30 in the morning.
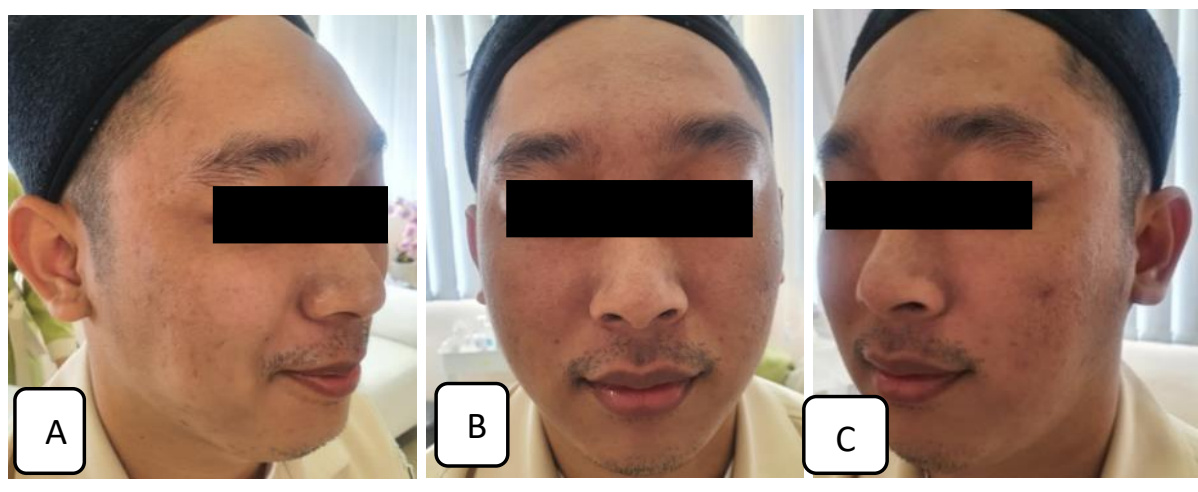

Figure 1. A; B; C. On facial regio revealed blackheads, whiteheads, papules, hyperpigmented macules, multiple atrophic acne scars.

\section{Discussion}

The diagnosis in this patient is based on history and physical examination findings. The patient is a 25 years old male with moderate comedonal acne vulgaris and severe atrophic post-acne scars. A person has a tendency of more than 3 times to suffer from acne if there is a main family member who suffers from acne. Trauma mechanical is a trigger factor for acne and aggravates the condition of acne, because trauma causes inflammation in the pathogenesis of acne. ${ }^{4,5}$ The patient has genetic factors in the family who tend to have acne and the patient's habit of holding his face and squeezing pimples causes acne to multiply with the formation of scars.

Microneedling radiofrequency has proven to be effective in the treatment of rolling type acne scars and boxcar scars. Microneedling radiofrequency devices work by creating a radiofrequency thermal zone without causing epidermal injury. After damage to the reticular dermis, long-term dermal remodeling, neoelastogenesis, and neocollagenogenesis result in dermal thickening. Microneedling radiofrequency combines microneedling with radiofrequency heat energy to increase collagen and elastin production. Microneedling radiofrequency is a minimally invasive treatment to reduce the appearance of scar tissue. 
Vejjabhinanta et al (Thailand, 2015) performed microneedling radiofrequency with atrophic acne scars with skin phototypes III-V who completed all treatment protocols. At the 6-month follow-up visit, most of the subjects (42.3\%) reported a 26-50\% improvement in the patients' acne scars. ${ }^{6,7}$

Several side effects of microneedling radiofrequency have been reported in various studies. Some of them are pain during the procedure, transient erythema and edema, post-inflammatory hyperpigmentation to scars in the form of discrete papules with linear patterns according to needle traces. The patient's side effect that occurred was facial edema which appeared 6 hours after the procedure and subsided the next day.6,8 The radiofrequency microneedling system has been shown to be effective in treating atrophic scars in darkskinned types with minimal risk of side effects. In the evaluation for 2 weeks after the procedure, this patient did not find any side effects, either complaints or clinically. In this case there was an improvement in the patient. Multiple sessions and further follow ups are needed for clinical improvement of the scar.

\section{Conclusion}

We report a case of 25 years old male patient with moderate comedonal acne vulgaris and severe atrophic post-acne scars who improved with a combination of topical preparations and microneedling radiofrequency procedures. In this case, we did not find any side effects on the patient.

\section{References}

1. Kesty K, Goldberg DJ. Radiofrequency microneedling for acne, acne scars, and more. Dermatological Reviews. 2020; 1: 33-37.

2. Min S, Park SY, Yoon JY, Kwon HH, Su DH. Fractional microneedling radiofrequency treatment for acne-related post-inflammatory erythema. Acta dermato-venereologica. 2016: 96(1): 87-91.

3. Goh C, Cheng C, Rather G, Zaenglein AL, Graber AM, Thiboutot DM, et al. Acne vulgaris. In: Kang S, Amagai M, Bruckner A, Enk A, Margolis D, McMichael A, et al. Fitzpatrick's Dermatology. 9th edition. New York: McGrawHill; 2019: 1391-1418.

4. Pudukadan D. Treatment of Acne Scars on Darker Skin Types Using a Noninsulated Smooth Motion, Electronically Controlled Radiofrequency Microneedles Treatment System. The American Society for Dermatologic Surgery. 2017; 43: S64-S69.

5. Park JY, Lee EG, Yoon MS, Lee HJ. The efficacy and safety of combined microneedle fractional radiofrequency and sublative fractional radiofrequency for acne scars in Asian skin. Journal of Cosmetic Dermatology. 2015; 15: 102-107.

6. Vejjabhinanta $\mathrm{V}$, Wanitphakdeedecha $\mathrm{R}$, Limtanyakul P, Manuskiatti W. The efficacy in treatment of facial atrophic acne scars in Asians with a fractional radiofrequency microneedle system. The Journal of the European Academy of Dermatology and Venereology. 2014; 28: 1219-1225.

7. Forbat E, Al-Niaimi. Fractional Radiofrequency Treatment in Acne Scars: Systematic review of current evidence. Journal of Cosmetic and Laser Therapy. 2016; 18(8): 442-447.

8. Weiner SF. Radiofrequency microneedling. Facial Plastic Surgery Clinics of North America. 2019; 27(3): 291-303. 